\title{
Imaging features of sentinel lymph node mapped by multidetector-row computed tomography lymphography in predicting axillary lymph node metastasis
}

Xiaochan Ou', Jianbin Zhu' ${ }^{1}$ Yaoming Qu' ${ }^{1}$ Chengmei Wang ${ }^{1}$, Baiye Wang ${ }^{1}$, Xirui Xu², Yanyu Wang ${ }^{1}$, Haitao Wen ${ }^{1}$, Andong Ma ${ }^{1}$, Xinzi Liu ${ }^{1}$, Xia Zou ${ }^{1}$ and Zhibo Wen ${ }^{1 *}$

\begin{abstract}
Introduction: Accurately assessing axillary lymph node (ALN) status in breast cancer is vital for clinical decision making and prognosis. The purpose of this study was to evaluate the predictive value of sentinel lymph node (SLN) mapped by multidetector-row computed tomography lymphography (MDCT-LG) for ALN metastasis in breast cancer patients.

Methods: 112 patients with breast cancer who underwent preoperative MDCT-LG examination were included in the study. Long-axis diameter, short-axis diameter, ratio of long-/short-axis and cortical thickness were measured. Logistic regression analysis was performed to evaluate independent predictors associated with ALN metastasis. The prediction of ALN metastasis was determined with related variables of SLN using receiver operating characteristic (ROC) curve analysis.
\end{abstract}

Results: Among the 112 cases, 35 (30.8\%) cases had ALN metastasis. The cortical thickness in metastatic ALN group was significantly thicker than that in non-metastatic ALN group ( $4.0 \pm 1.2 \mathrm{~mm}$ vs. $2.4 \pm 0.7 \mathrm{~mm}, P<0.001)$. Multilogistic regression analysis indicated that cortical thickness of $>3.3 \mathrm{~mm}(\mathrm{OR} 24.53,95 \% \mathrm{Cl} 6.58-91.48, P<0.001)$ had higher risk for ALN metastasis. The best sensitivity, specificity, negative predictive value(NPV) and AUC of MDCT-LG for ALN metastasis prediction based on the single variable of cortical thickness were $76.2 \%, 88.5 \%, 90.2 \%$ and 0.872 (95\% $\mathrm{Cl} 0.773-0.939, P<0.001)$, respectively.

Conclusion: ALN status can be predicted using the imaging features of SLN which was mapped on MDCT-LG in breast cancer patients. Besides, it may be helpful to select true negative lymph nodes in patients with early breast cancer, and SLN biopsy can be avoided in clinically and radiographically negative axilla.

Keywords: Breast cancer, Multidetector-row computed tomography lymphography, Sentinel lymph node, Axillary lymph node metastasis, Cortical thickness

*Correspondence: zhibowen@163.com

${ }^{1}$ Department of Radiology, Zhujiang Hospital, Southern

Medical University, 253 Gongye Middle Avenue, Haizhu District,

Guangzhou 510282, Guangdong, China

Full list of author information is available at the end of the article

\section{Background}

Recently, a large population-based study reported that an increasing global incidence of breast cancer and there are about 645,000 premenopausal and 1.4 million postmenopausal breast cancer cases were diagnosed worldwide [1]. Breast cancer may often be associated with axillary 
lymph node (ALN) metastases, and ALN status is one of the most important predictor of overall recurrence and survival in patients with breast cancer. Accurate assessment of ALN disease burden is vital in staging breast cancer, which guides multidisciplinary treatment decision making nowadays [2,3]. Sentinel lymph node (SLN) biopsy (SLNB) is now widely used as the gold standard for axillary staging in clinically node-negative breast cancer in National Comprehensive Cancer Network $(\mathrm{NCCN})$ guidelines [4], which reduces the complications of lymphedema and other arm morbidity to a certain extent compared with axillary lymph node dissection (ALND) [5].

Assessment of ALN disease involvement is currently considered to be the most important role of axillary imaging [2]. In clinical practice, conventional imaging techniques for ALN status assessment include mammography (MMG), ultrasonography (US) and magnetic resonance imaging (MRI) [6]. Axillary level I lymph nodes (LNs) are visible at routine MMG in 50\% of patients [7], but limited visualization makes it unreliable to assess ALN [8]. US is the primary modalities to evaluate ALN, nonetheless, results may vary according to operator mainly [9]. A retrospective study has shown that US cannot distinguish between localized and advanced ALN disease when the result is positive [10], which may lead to over-treatment under the Z0011 standard since 40-70\% patients had the SLN as the only site of nodal metastasis [11]. Indeed, some medical institution have abandoned preoperative axiilary US in patient with negative finding on physical examination to avoid triaging those women with positive ALN directly to ALND [12, 13]. ALNs with standard breast MRI revealed comparable performance to that of US [14]. Although MRI has dedicated breast coil, its ability to show the complete axilla is limited, and cardiac motion artifacts can occasionally block ALN, especially in leve II and III LNs [15]. The indications of MRI mainly include clinically staging of breast cancer, screening of high-risk populations and efficacy evaluation of neoadjuvant chemotherapy [16], However, it may be more commonly used for imaging and detection of primary cancers. In addition, MRI is time-consuming and expensive, making it not widely used in clinical practice [15].

Many previous studies have shown that multidetector-row computed tomography (MDCT) can be used to evaluate regional LNs [17-19]. High-resolution helical CT could show not only eccentric or irregular cortical thickening in metastasis LN, but detect extracapsular LN extension [20], which is a potent imaging tool for predicting ALN metastasis. Besides, Chest CT can be used to evaluate axillary nodes in patients with advanced breast disease and showed better diagnostic value for visualizing level III LNs, interpectoral nodes, and extensive nodal involvement [21]. Chen et al. had shown that MDCT is an effective imaging tool for predicting ALN metastasis [22] Preoperative multidetector-Row computed tomographic lymphography (MDCT-LG) can be used to locate SLN in patient with early breast cancer [23], because the tumor cells almost always invade ALN sequentially and usually starting with SLN [24, 25]. Moreover, studies have shown that MDCT-LG in addition to being reliable navigation instrument for SLN biopsy (SLNB) that helps surgeons quickly find the SLN, reduce surgical time and improve the accuracy of the SLNB, it may also be helpful in determining the scope of LNs cleaning [26], The anatomic morphology of lymphatic vessels and SLNs can also be understood preoperatively, which is beneficial to improve the success rate of SLNB surgery [27]. Nakagawa and Ashiba et al. used MDCT-LG to diagnosed SLN metastasis before surgery as well, But neither took into account the thickness of the LN cortex [28,29].

The purpose of our study was to assess the value of MDCT-LG determined SLN imaging features in predicting ALN status.

\section{Methods and materials \\ Patients enrollment}

The study was approved by the local Institutional Review Board, and because its nature of retrospective study, the requirement of obtaining informed consent was waived. Breast cancer patients who underwent MDCT-LG and had a clear display of SLNs and subsequent surgery between January 2019 and March 2021 were included in our study. The inclusion criteria were: (1) Women aged 18 years or older; (2) Images can clearly locate sentinel lymph nodes; (3) First diagnosis of breast cancer; (4) Breast cancer was pathologically confirmed by surgery or biopsy; The exclusion criteria included: (1) patients with incomplete pathological results; (2) patients were diagnosed with distant metastasis; (3) patients who had undergone tumor resection or neoadjuvant chemotherapy prior to MDCT-LG; (4) Lactation patient; All the patients received surgery for axillary staging, included SLNB, ALND or both. The number of lymph nodes resected were recorded, with subsequent pathological examination and confirmation by two pathologists. Clinicopathological characteristic (including age, sex, tumor size, histology, and ALN metastasis status) were collected.

\section{MDCT-LG}

All images were obtained with a 64-detector row CT scanner (Brilliance 64, Philips, Netherlands). Patients were placed in a supine position with their arms positioned in a cranial direction. Local anesthesia was 
performed by subcutaneous injection of $4 \mathrm{ml}$ lidocaine $(0.02 \mathrm{~g} / \mathrm{ml})$ with a $5-\mathrm{ml}$ disposable sterile syringe (Zhejiang Jinghuan Medical Supplies Co., LTD), followed by, intradermal injection of $1-\mathrm{ml}$ iopamidol $(370 \mathrm{mg} / \mathrm{ml}$, Obilol, Shanghai, Bolaik Xini Pharmaceutical Co.,LTD) of intradermal injection at 3, 6, 9 and 12 o'clock of areola, respectively. Finally contiguous 1 -mm-thick images that included the breast and axilla were obtained after gently massaging the injection site for about $30 \mathrm{~s}$. The CT scanning was performed with the following parameters: $120 \mathrm{kV}$ and $250 \mathrm{~mA}$, field view of $32 \mathrm{~cm} \times 32 \mathrm{~cm}$, matrix of field of view a $512 \times 512$, a section spacing of $1 \mathrm{~mm}$. The number of sections were adjusted for each individual to ensure coverage of breast and axillary areas. After the CT scanning, a 3D reconstruction was performed on the Philips IntelliSpace Portal to determine the SLN and its location. The identification of the SLN was completed by a breast surgeon and a radiologist with 3 years of experience.

\section{MDCT-LG evaluation of the nodal status}

MDCT-LG Evaluation was performed on the Philips IntelliSpace Portal platform with 3D resconstruction. Two radiologists (with 3 years of experience of breastradiology) reviewed the images and measured the relevant parameters. For those with more than one SLNs, the largest one would have been selected and measured. The recorded parameters include: (1) the shape of SLN; (2) long-axis diameter; (3) short-axis diameter; (4) Ratio of long-/short-axis; (5) cortical thickness. The data measurement method is shown in Fig. 1. According to the pathological results of ALN, they were divided into metastatic group and non-metastatic group. The relationship of lymph nodes to pectoralis minor divides the axillary lymph nodes into levels I, II and III. The lymph nodes located lateral to the lateral border of the pectoralis minor are level I nodes. The axillary located between the medial and lateral boundaries of the pectoralis minor or interpectoral (Rotter's) lymph nodes are level II nodes. The lymph nodes located medial to the medial margin of the pectoralis minor muscle and inferior to the clavicle are level III nodes [30].

\section{Pathology}

Primary breast cancer is divided into invasive breast cancer and ductal carcinoma in situ according to pathology. T1 disease is defined as a primary tumor size of less than or equel to $20 \mathrm{~mm}$; T2 disease is defined as a primary tumor size of more than $20 \mathrm{~mm}$ but less than or equal to $50 \mathrm{~mm}$; T3 disease is defined as a primary tumor size of more than $50 \mathrm{~mm}$ [30]. The pathologist diagnosed each lymph node as benign or malignant.

\section{Statistical analysis}

Continuous and categorical variables were analyzed with Mann Whitney U-test and X2-test respectively. Logistic regression analysis (stepwise) was performed to evaluate the variates associated with ALN metastasis so that significant or marginal significant factors $(P<0.05)$ could be identified. Receiver operating characteristic (ROC) curve
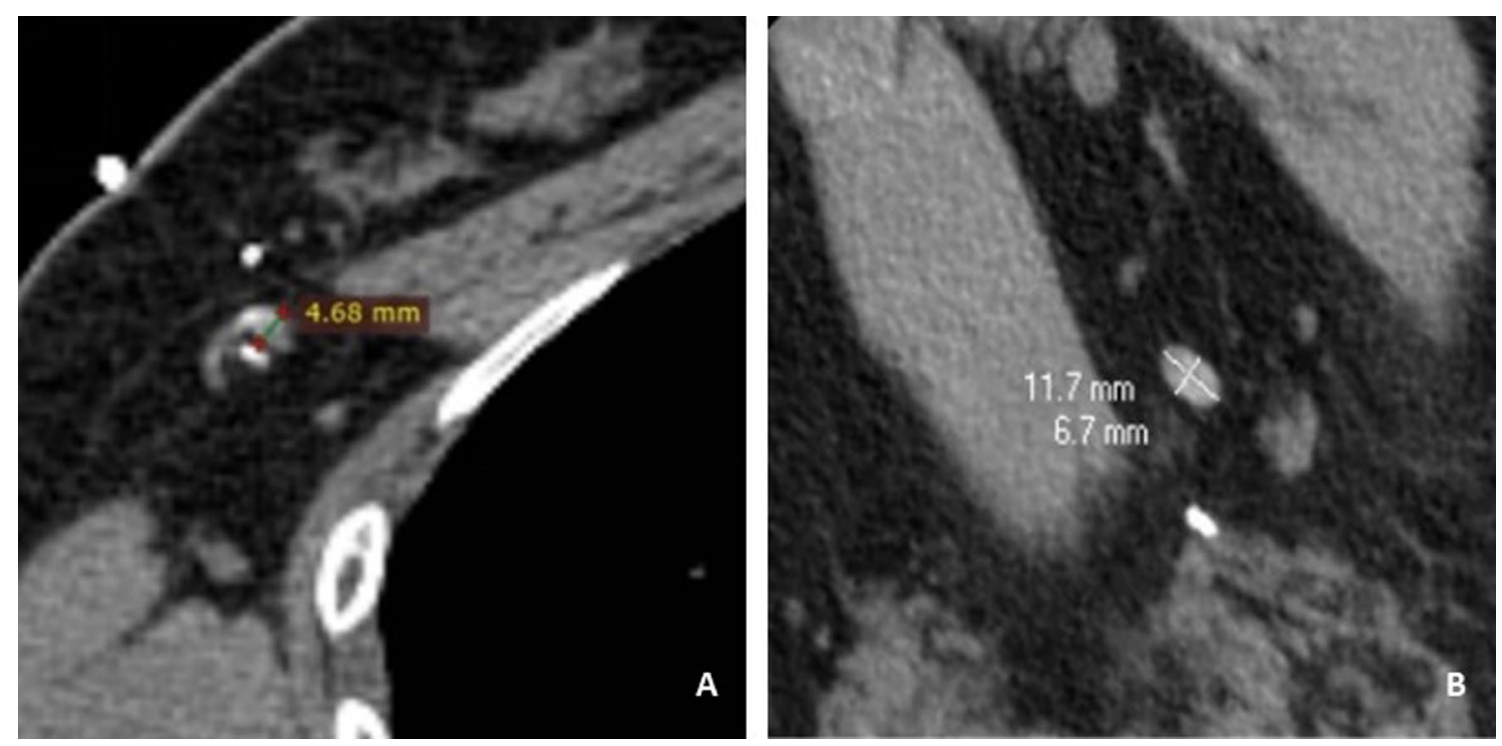

Fig. 1 Methods of measuring long and short diameter of sentinel lymph nodes and cortical thickness. A The maximum layer of cortical thickness of selected sentinel lymph nodes was measured in RadiAnt DICOM Viewer (64-bit). B The maximum long diameter and maximum short diameter of the maximum section of the selected sentinel lymph node were measured in Philips IntelliSpace portal 
analysis was performed to evaluate the MDCT variates for predicting ALN metastasis. The area under the ROC curve (AUC) was evaluated for diagnostic ability. The optimal cutoff value was based on the ROC curve with Youden's J statistic (J), J= sensitivity + specificity -1 . $P<0.05$ was considered statistically significant. Statistical analysis was performed using SPSS statistical software (version 18.0, SPSS Inc., Chicago, IL, USA) and MedCalc Statistical Software (version 19.5.6, MedCalc Software bvba, Ostend, Belgium).

\section{Result}

Patients characteristic

A total of 149 cases underwent MDCT-LG and had a clear display of SLNs. Thirty-seven patients were excluded because of: (1) incomplete pathological results $(\mathrm{n}=14)$; (2) Distant metastasis $(\mathrm{n}=3)$; (3) tumor resection or neoadjuvant chemotherapy prior to MDCT-LG $(\mathrm{n}=19)$; (4) during breast feeding $(\mathrm{n}=1)$. Finally, 112 cases were enrolled in the study. A total of 108 cases (96.4\%) of SLNs identified by preoperative MDCT-LG were at the level I, and 4 cases (3.6\%) were at the level II of the axillary clinical group. Totally have 87 cases $(78.3 \%)$ performed SLNB, 24 (20.0\%) cases performed both SLNB and ALND, and 1case (1.7\%) performed ALND directly.

All patients were female, with a mean age of 49.7 years (range 23-76 years). Tumor size was measured based on gross specimen after surgery, with a median tumor size of $14.5 \mathrm{~mm}$ (range 7-80 $\mathrm{mm}$ ). In line with the American Joint Committee on Cancer (AJCC) 7-stage system, 53 (47.3\%) were stage T1, 53 (47.3\%) were stage T2, and 6 (5.4\%) were stage T3. Pathological examination revealed that 108 cases $(96.4 \%)$ were invasive breast cancer and 4 cases were ductal carcinoma in situ (DCIS). All patients underwent axillary surgery, 35 (30.8\%) cases with ALN metastasis, including 22 cases (19.6\%) with SLN metastasis alone, 12 cases (10.7\%) with both SLN and non-SLN metastasis, and 1 case $(0.9 \%)$ with non-SLN metastasis alone (Table 1).

\section{MDCT-LG variables of sentinel lymph nodes}

As shown in Table 2, the long-axis diameter and shortaxis diameter of metastatic SLN were significantly longer than that of non-metastatic SLN $(14.6 \pm 6.2 \mathrm{~mm}$ vs.11.1 $\pm 3.3 . \mathrm{mm}, \quad P<0.001 ; \quad 10.7 \pm 4.2 \mathrm{~mm} \quad$ vs. $6.2 \pm 2.0 \mathrm{~mm}, P<0.001)$. The average ratio of long-/ short-axis of metastatic SLN was significantly shorter than that of non-metastatic SLN $(1.4 \pm 0.3$ vs. $1.7 \pm 0.3$, $P=0.002)$. The thickness of cortex in metastatic SLN was significantly thicker than that in non-metastatic SLN ( $4.0 \pm 1.2 \mathrm{~mm}$ vs. $2.4 \pm 0.7 \mathrm{~mm}, P<0.001)$. No significant
Table 1 Patient characteristics

\begin{tabular}{lcc}
\hline Characteristics & Median & $\begin{array}{l}\text { No. of } \\
\text { patients/ } \\
\text { SLN (\%) }\end{array}$ \\
\hline Female & $112(100.0)$ \\
Age(years) & \\
Tumor size $(\mathrm{mm})$ & $49.7(23-76)$ & \\
$T \leq 20 \mathrm{~mm}$ & $24.5(7-80)$ & $53(47.3)$ \\
20 mm $<T \leq 50$ mm & & $53(47.3)$ \\
$T>50$ mm & $6(5.4)$ \\
Histology & & $108(96.4)$ \\
Invasive breast cancer & $4(3.6)$ \\
DCIS & $35(100.0)$ \\
ALN metastasis & $20(17.9)$ \\
Only SLN & $3(2.8)$ \\
Only non-SLN & $12(10.7)$ \\
Both SLN and non-SLN & & \\
\hline
\end{tabular}

difference was found in the shape of $\operatorname{SLN}(P=0.352)$ (Table 2).

\section{Univariate and multivariate analysis}

Univariate analysis showed that long-axis diameter, short-axis diameter, ratio of long-/short-axis, cortical thickness were significantly associated with ALN metastasis. Multivariate analysis demonstrated that cortical thickness were independent predictors for ALN metastasis (OR 24.53, 95\% CI 6.58-91.48, $P<0.001$ ) (Table 3).

\section{Comparison of axillary lymph nodes metastasis prediction performance}

The ROC based on univariate analysis showed that $13.9 \mathrm{~mm}$ was the optimal cut-off value of long-axis diameter, with an AUC, sensitivity and specificity were of 0.725 (95\% CI $0.633-0.805, P<0.001), 54.3 \%$ and $87.0 \%$, respectively. The optimal cut-off value of short-axis diameter was $9.1 \mathrm{~mm}$, with an AUC, sensitivity and specificity were 0.801 (95\% CI $0.715-0.870$, $P<0.001), 62.8 \%$, and $92.2 \%$, respectively. The optimal cut-off value of ratio of long-/short-axis was 1.7 , with an AUC, sensitivity and specificity were of 0.679 (95\% CI $0.584-0.764, P=0.004), 82.7 .0 \%$ and $46.8 \%$, respectively. The optimal cut-off value of cortical thickness was $3.3 \mathrm{~mm}$, with an AUC, sensitivity and specificity were of 0.872 (95\% CI $0.773-0.939, P<0.001), 76.2 \%$ and $88.5 \%$, respectively. The short-axis diameter had the highest specificity, $92.2 \%$. The ratio of long-/shortaxis had the highest sensitivity, $82.9 \%$. (Tables 3,4 and Fig. 2). The AUC of cortical thickness and short-axis diameter were larger than that of long-axis diameter, 
Table 2 Comparison of SLN metastasis with MDCT-LG variables

\begin{tabular}{|c|c|c|c|c|}
\hline \multirow[t]{2}{*}{ Characteristic } & \multicolumn{3}{|c|}{ ALN metastasis } & \multirow[t]{2}{*}{$P$ value } \\
\hline & & Positive $(n=35)$ & Negative $(n=77)$ & \\
\hline Long-axis diameter(mm) & $11.6 \pm 4.8$ & $14.6 \pm 6.2$ & $11.1 \pm 3.3$ & $<0.001$ \\
\hline Short-axis diameter(mm) & $7.0 \pm 3.4$ & $10.7 \pm 4.2$ & $6.2 \pm 2.0$ & $<0.001$ \\
\hline Ratio of long-/short-axis & $1.6 \pm 0.3$ & $1.4 \pm 0.3$ & $1.7 \pm 0.3$ & 0.002 \\
\hline Cortical thickness(mm) & $2.6 \pm 1.1$ & $4.0 \pm 1.2$ & $2.4 \pm 0.7$ & $<0.001$ \\
\hline Shape & & & & 0.352 \\
\hline Oval & 92 & 27 & 65 & \\
\hline Round & 20 & 8 & 12 & \\
\hline
\end{tabular}

Table 3 Univariate and multivariate logistic regression analysis for SLN metastasis

\begin{tabular}{|c|c|c|c|c|c|}
\hline \multirow[t]{2}{*}{ Variable } & \multirow[t]{2}{*}{ Cut-off value } & \multicolumn{2}{|c|}{ Univariate analysis } & \multicolumn{2}{|l|}{ Univariate analysis } \\
\hline & & OR $(95 \% \mathrm{Cl})$ & $P$ value & OR $(95 \% \mathrm{Cl})$ & $P$ value \\
\hline \multirow[t]{2}{*}{ Long-axis diameter(mm) } & $>13.9$ & $7.09(2.78-18.13)$ & $<0.001$ & - & 0.249 \\
\hline & $\leq 13.9$ & - & - & - & - \\
\hline \multirow[t]{2}{*}{ Short-axis diameter(mm) } & $>9.1$ & $20.03(6.81-58.92)$ & $<0.001$ & - & 0.630 \\
\hline & $\leq 9.1$ & - & - & - & - \\
\hline \multirow[t]{2}{*}{ Ratio of long-/short-axis } & $\leq 1.7$ & $4.24(1.58-11.38)$ & 0.004 & - & 0.310 \\
\hline & $>1.7$ & - & - & - & - \\
\hline \multirow[t]{2}{*}{ Cortical thickness(mm) } & $>3.3$ & $24.53(6.58-91.48)$ & $<0.001$ & $23.50(6.27-88.10)$ & $<0.001$ \\
\hline & $\leq 3.3$ & - & - & - & - \\
\hline
\end{tabular}

Table 4 Optimal cut-off values for diagnosis of SLN metastasis with MDCT-LG

\begin{tabular}{|c|c|c|c|c|c|c|}
\hline MDCT-LG variates & $\begin{array}{l}\text { Optimal cut-off } \\
\text { value }\end{array}$ & Sensitivity (\%) & Specificity (\%) & AUC & PPV (\%) & NPV (\%) \\
\hline Long-axis diameter (mm) & $>13.9$ & 54.3 & 87.0 & 0.725 & 78.6 & 84.5 \\
\hline Short-axis diameter (mm) & $>9.1$ & 62.9 & 92.2 & 0.801 & 64.3 & 79.8 \\
\hline Ratio of long-/short-axis (mm) & $\leq 1.7$ & 82.9 & 46.8 & 0.679 & 82.9 & 46.8 \\
\hline Cortical thickness (mm) & $>3.3$ & 76.2 & 88.5 & 0.872 & 72.7 & 90.2 \\
\hline
\end{tabular}

and it was statistically significant. The comparison of AUC between cortical thickness and short-axis diameter was not statistically significant, $P=0.059$. The ratio of long-/short-axis and cortical thickness had the highest positive predictive value $(82.7 \%)$ and negative predictive value $(90.2 \%)$, respectively.

According to the above results of univariate analysis, three variables with $P<0.001$ were selected for combination and their respective AUC were compared. The three variables were long-axis diameter, short-axis diameter and cortical thickness. Combinedanalysis indicated that combination of both or three of the variables did not improve diagnostic specificity or sensitivity. As shown in Tables 4 and 5, the results of combination of long-axis diameter and short-axis diameter were same as those of single variable of shortaxis diameter, they had the same AUC, sensitivity, and specificity. The AUC, sensitivity, and specificity of the three combined variables were the same as those of the single variable of cortical thickness.

\section{Discussion}

Our study is that we selected the MDCT-LG mapped SLN as the study subject, and using it, we can not only predict ALN metastasis, but may help select a portion of patients who can be exempted from SLNB and make treatment more personalized. 


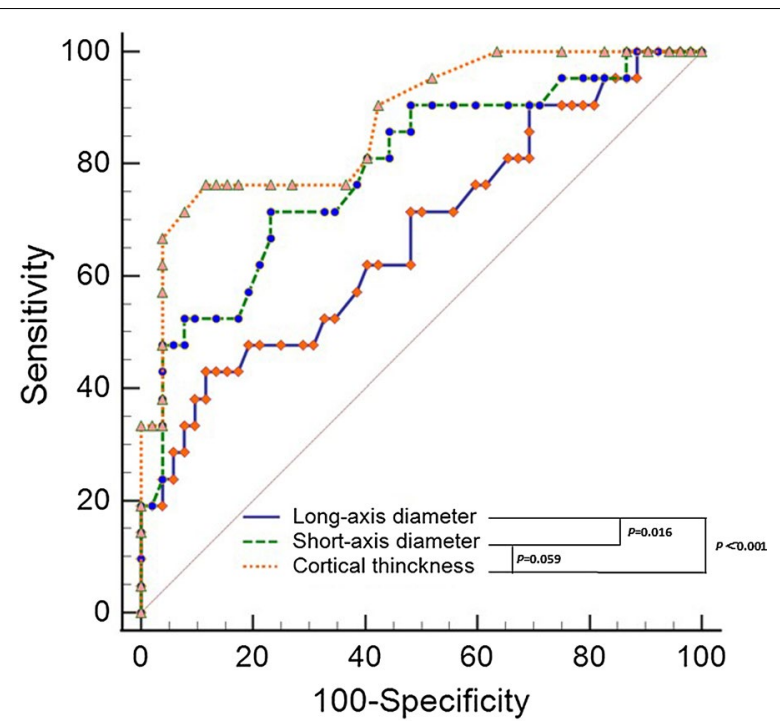

Fig. 2 Comparison of areas under the curve of long-axis diameter, short-axis diameter, and cortical thickness

Chen et al. showed MDCT could predict ALN metastasis by observing and measuring the biggest LN in the axilla [22], but it is not clear whether the largest lymph node is the SLN. Compared with their study, we mapped SLN prior to operation, which helps clinicians determine the surgical approach of SLNB, thus shortening the surgical time and improving the accuracy of SLNB. Besides, knowing the location of SLN in advance could help surgeons to determine the approximate range of LNs resection, which is especially significant for patients with SLN at level II or III, and would not lead to SLN miss.

In terms of diagnosing lymph node status, this study demonstrated that LN metastasis of breast cancer is usually orderly, and SLN usually located at level I, which support the results of Kalli et al., namely, breast cancer cells spread progressively and orderly along the lymphatic drainage system, with level I first metastasis in most cases [30]. Both long-axis diameter and short-axis diameter of the metastasis SLN are significantly longer than that of the non-metastasis SLN, which is consistent with previous research results [31,32]. As for the short-axis diameter, it is recommended to distinguish between benign and malignant LNs in the RECIST 1.1 [33]. In line with previous CT and MRI studies, the mean ratio of long-/ short-axis of metastasis lymph node was lower than that of non-metastatic one [17, 34].

For the long-axis diameter, the ROC showed that $13.9 \mathrm{~mm}$ was the optimal cut-off value, and our result was close to that of Chen et al. [22], whose was $14.5 \mathrm{~mm}$, but their statistical analysis indicated that long-axis diameter was not a statically significant factor, whereas, our study suggested that long-axis diameter is one of the factor to identify metastatic from non-metastatic LNs. Whether evaluating axillary or cervical nodes, short-axis diameter is commonly used by radiologist because it is a repeatable measure to predict metastasis [34]. Different sites have corresponding drainage LNs, but the recommended diagnostic criteria for short-axis diameter vary from site to site $[17,19,35-37]$. At present, there is no standard value about the optimal short-axis diameter of ALN. Our study indicated that $9.1 \mathrm{~mm}$ was the best short-axis diameter cut-off value. Previous researchers have used short-axis diameter $\geq 10 \mathrm{~mm}$ as the cut-off value for diagnosing metastatic ALN [31, 37]. In addition, the ratio of long-/short-axis on the image could also indicate ALN involvement. Liu et al.demonstrated that combination of smaller size and lymph node axial ratio can Improve CT detection sensitivity for nodal metastases in oesophageal cancer [38]. Both the two indexes of our study above are similar to those of Chen et al. [22], whose optimal cut-off values of the short-axis diameter and ratio of long-/shortaxis are $9.5 \mathrm{~mm}$ and 1.7 respectively, and the statistical analysis results showed that there are significant differences. Although the long-axis diameter, short-axis diameter and ratio of long-/short-axis were not independent predictors, they were statistically significant in Univariate analysis, which may be caused by the insufficient number of cases.

We measured the cortical thickness of the SLN as well. Although cortical thickening is often associated with reactive lymph node hyperplasia, prospective studies of cortical thickness of ALNs in preoperative breast cancer patients have shown that the incidence of malignancy increases in proportion to cortical thickness [39]. Because malignant cells enter the LN through the subcapsular sinus in the form of afferent lymphoid deposits

Table 5 AUC comparison of combinatiorial factors

\begin{tabular}{llll}
\hline MDCT-LG variates & Sensitivity (\%) & Specificity (\%) & AUC \\
\hline Short-axis diameter + Long-axis diameter & 62.3 & 92.2 & 0.801 \\
Long-axis diameter + Cortical thickness & 76.2 & 88.5 & 0.872 \\
Short-axis diameter + Cortical thickness & 76.2 & 88.5 & 0.872 \\
Combination of three variables & 76.2 & 88.5 & 0.872 \\
\hline
\end{tabular}


(local diffusion), where they grow and eventually replace locally normal lymph node structures [40]. In fact, cortical thickening of metastatic lymph nodes is often found in axillary images [39, 41]. Our results showed a significant difference in cortical thickness between the metastatic and non-metastatic groups. Moreover, cortical thickness was an independent predictor of ALN metastasis. In our study, the optimal cut-off value of cortical thickness was $3.3 \mathrm{~mm}$. This is different from the results of Chen and Imai et al. whose results are $3 \mathrm{~mm}[22,31]$.

Ultrasound is the first choice for the assessment of ALNs and imaging guided lymph node interventional therapy [42]. Marino et al. reported sensitivity and specificity of ultrasound, standard MRI, and PET/CT in assessing ALN: $87 \%$ and $53 \%$ to $97.3 \%, 70 \%$ and $90 \%$, $64 \%$ and $93 \%$, respectively [42]. In this study, the sensitivity, specificity and AUC of the single variable of cortical thickness were $76.2 \%, 88.5 \%$ and 0.872 , respectively. In terms of sensitivity, our results indicate that the sensitivity is not as good as that of ultrasound, but slightly better than that of PET/CT and standard MRI. In terms of specificity, our results are very close to PET/CT, but slightly inferior to MRI. Compared with standard MRI, using dedicated axillary protocols can increase the sensitivity and specificity to $84 \%$ and $95 \%$, respectively, but they require additional scanning time and are not feasible in clinical practice [43]. Overall, SLN measured with more parameters, including cortical thickness $<3.3 \mathrm{~mm}$, Short-axis diameter $<9.1 \mathrm{~mm}$, and Ratio of long-/shortaxis $\geq 1.7$, were more likely to be negative lymph nodes and vice versa (Figs. 3, 4). In our view, when the results tend to be negative and the volume of non-SLNs are smaller than that of SLN, it can even be considered to omit SLNB in combination with the image characteristics, pathological grade and biological behavior of the lesion itself [44]. Because $5-8 \%$ of patients still develop lymphedema after the SLNB, and $60 \%$ of newly diagnosed breast cancer patients are pathologically lymph node negative, these patients do not benefit from it [45]. Furthermore, Data from Felix Jozsa et al. meta-analysis support the omission of SLNB in clinically and radiographically negative axilla [46]. Due to the object of our study was SLN, we can consider direct ALND for these cases if MDCT-LG indicates skip metastasis when nonSLN metastasis is obvious.

Our study focused only on SLN, but it is still useful for preoperative assessment of ALN status, especially the status of SLN. For those lymph nodes with typical cortical structure, we believe that it may be possible to select patients who can avoid SLNB. Though it is still not a replacement for the SLNB now, some scholars considered that the removal of these LNs is not a necessary procedure to prevent the spread of the disease in recent years and axillary surgery for breast cancer patients has tended to be downgraded [47]. Moreover, we want to emphasize that the images may be affected by uncertainties, and in these cases, fuzzy preprocessing techniques should be used, because it can enhance the contrast of the image, make the image more clear, the measured value will be more accurate $[48,49]$.

It is hoped that future prospective clinical trials will confirm these results, and that better scanning methods and parameters will be developed to assess ALN and accurately select patients with direct ALND and those who are exempt from SLNB. For those cases that are difficult to determine, the SLNB is performed first

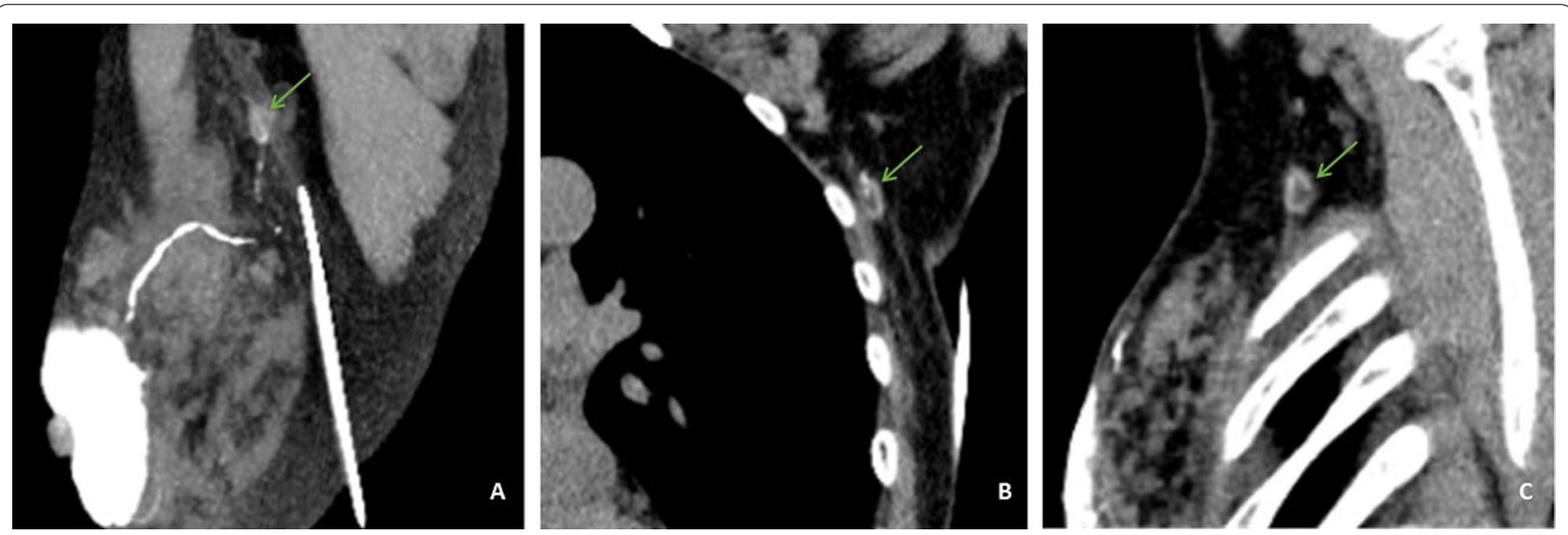

Fig. 3 Representative multidetector-row computed tomography Lymphography (MDCT-LG) images of a 58-year-old woman with left primary breast cancer (stage pT2N0, Non-specific invasive breast cancer). A The MDCT-CT 3D reconstruction image showed an sentinel lymph node was in level I. B, C Sagittal and coronal MDCT-LG image showed this lymph node with oval shape, a long-axis diameter of about $12.2 \mathrm{~mm}$, and a short-axisdiameter of about $5.4 \mathrm{~mm}$ (green arrows) and a cortical thickness about $2.2 \mathrm{~mm}$ (green arrows). The patient underwent sentinel lymph node biopsy, five lymph nodes were removed and proved to be pathologic negative 

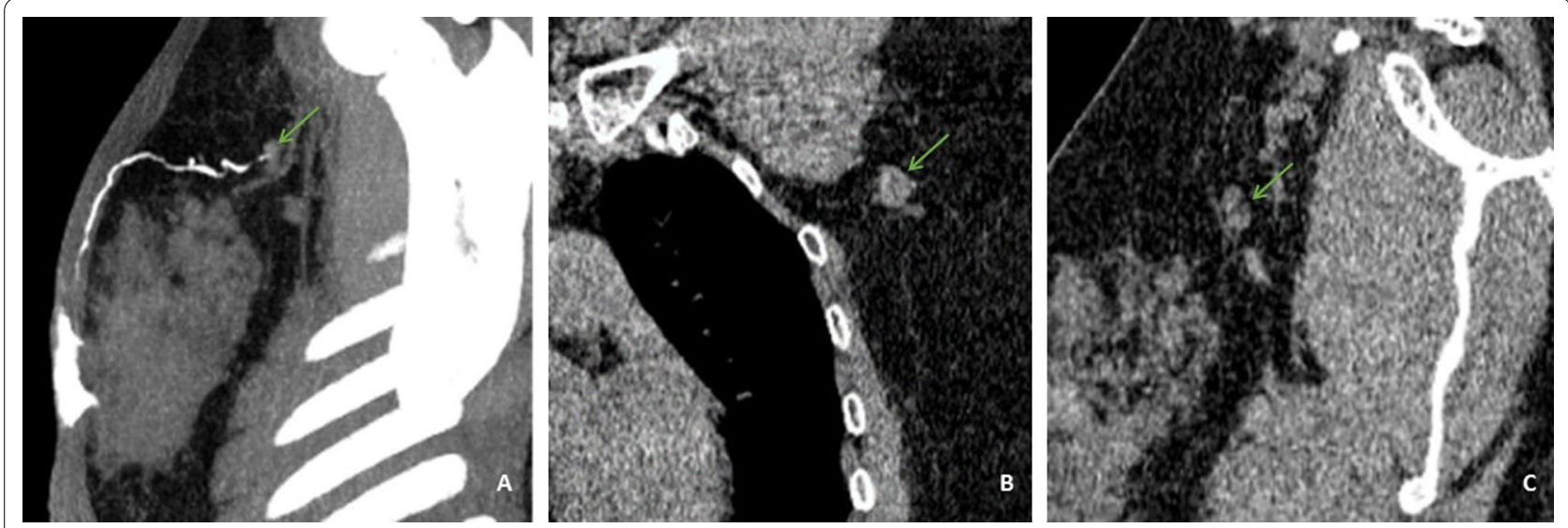

Fig. 4 Representative multidetector-row computed tomography lymphography (MDCT-LG) images of a 59-year-old woman with left primary breast cancer (stage pT3N1, Non-specific invasive breast cancer). A The MDCT-CT 3D reconstruction image showed an sentinel lymph node was in level I. B, C Sagittal and coronal MDCT-LG image showed this lymph node with oval shape, a long-axis diameter of about $13.3 \mathrm{~mm}$, and a short-axisdiameter of about $11.1 \mathrm{~mm}$ (green arrows) and a cortical thickness about $4.3 \mathrm{~mm}$ (green arrows). The patient underwent both sentinel lymph node biopsy and axillary lymph node dissection, a total of eighteen lymph nodes were removed, and sentinel lymph node biopsy removed seven lymph nodes, four of which proved to be pathologic positive

and then according to the Z0011 standard. Limitations of the study include: first, there may be biases due to the difficulty in making paired comparisons between MDCT imaging and pathologic findings in each lymph node. Second, this is a retrospective study with a relatively small sample size from a single institution, which may compromise the representativeness of the study.

\section{Conclusion}

SLN mapped by MDCT-LG has the potential to predict ALN metastasis. Cortical thickness of SLN in MDCTLG was an independent predictor of ALN metastasis. The optimal cut-off value of cortical thickness for predicting metastatic ALN was $3.5 \mathrm{~mm}$. It may possible to avoid SLNB in patients with clinically and radiographically negative axilla.

\section{Abbreviations \\ ALN: Axillary lymph node; ALND: Axillary lymph node dissection; MRI: Mag- netic resonance imaging (MRI); MMG: Mammography; MDCT-LG: Multide- tector-row computed tomography lymphography; ROC: Receiver operating characteristic; SLN: Sentinel lymph node; SLNB: Sentinel lymph node biopsy; US: Ultrasonography.}

\section{Acknowledgements}

Xiaochan Ou: Analysis and interpretation of data; Drafting of the manuscript; Review the of the manuscript for important intellectual content in the manuscript, and finally determine the submitted version. Jianbin Zhu and Yaoming Qu: Review of the manuscript for important intellectual content, and linguistic revision. Zhibo Wen: Review of the manuscript for important intellectual content and final approval of the version to be submitted.

\section{Authors' contributions}

$\mathrm{XO}, \mathrm{JZ}$ and $\mathrm{YQ}, \mathrm{CW}$ wrote the main manuscript text. BW, $\mathrm{XX}$, and $\mathrm{YW}$ prepared Figs. 1, 2, 3 and 4. HW, AM and XL, XZ prepared Tables 1, 2, 3, 4 and 5. All authors read and approved the final manuscript.

\section{Funding}

This research did not receive any specific grant from funding agencies in the public, commercial, or not-for-profit sectors.

\section{Availability of data and materials}

The datasets generated or analyzed in the current study are not publicly available because of patient privacy protection, but are available from the corresponding author upon reasonable request.

\section{Declarations}

\section{Ethics approval and consent to participate}

The study was carried out in accordance with the guidelines of the Declaration of HelsinkiThis retrospective and it was approved by the local institutional ethics committee (approval number: 2020-JS-026-01), and the requirement of obtaining informed consent was waived.

\section{Consent for publication}

The consent of all authors has been obtained.

\section{Competing interests}

No actual or potential conflict of interest exists.

\section{Author details}

'Department of Radiology, Zhujiang Hospital, Southern Medical University, 253 Gongye Middle Avenue, Haizhu District, Guangzhou 510282, Guangdong, China. ${ }^{2}$ Department of Breast Surgery, Zhujiang Hospital, Southern Medical University, 253 Gongye Middle Avenue, Haizhu District, Guangzhou 510828, Guangdong, China.

Received: 30 October 2021 Accepted: 26 November 2021

Published online: 15 December 2021 


\section{References}

1. Heer E, et al. Global burden and trends in premenopausal and postmenopausal breast cancer: a population-based study. Lancet Glob Health. 2020;8(8):e1027-37.

2. Chang JM, et al. Axillary nodal evaluation in breast cancer: state of the art. Radiology. 2020;295(3):500-15.

3. Caudle AS, Cupp JA, Kuerer HM. Management of axillary disease. Surg Oncol Clin N Am. 2014;23(3):473-86.

4. Gradishar WJ, et al. NCCN guidelines insights: breast cancer, version 1.2017. J Natl Compr Canc Netw. 2017;15(4):433-51.

5. Yen TWF, et al. Prevalence and consequences of axillary lymph node dissection in the era of sentinel lymph node biopsy for breast cancer. Med Care. 2018;56(1):000-000.

6. Lowes $\mathrm{S}$, et al. Evolving imaging techniques for staging axillary lymph nodes in breast cancer. Clin Radiol. 2018;73(4):396-409.

7. Shetty MK, Carpenter WS. Sonographic evaluation of isolated abnormal axillary lymph nodes identified on mammograms. J Ultrasound Med. 2004;23(1):63-71.

8. Valente SA, et al. Accuracy of predicting axillary lymph node positivity by physical examination, mammography, ultrasonography, and magnetic resonance imaging. Ann Surg Oncol. 2012;19(6):1825-30.

9. Hooley RJ, Scoutt LM, Philpotts LE. Breast ultrasonography: state of the art. Radiology. 2013;268(3):642-59.

10. Schipper RJ, et al. Axillary ultrasound for preoperative nodal staging in breast cancer patients: is it of added value? The Breast. 2013;22(6):1108-13.

11. Grube BJ, Giuliano AE. Observation of the breast cancer patient with a tumorpositive sentinel node: implications of the ACOSOG Z0011 trial. Semin Surg Oncol. 2001;20(3):230-7.

12. Louie RJ, et al. Trends in surgical axillary management in early stage breast cancer in elderly women: continued over-treatment. Ann Surg Oncol. 2020:27(9):3426-33.

13. Wallis MG, Kilburn-Toppin F, Taylor-Phillips S. Does preoperative axillary staging lead to overtreatment of women with screen-detected breast cancer? Clin Radiol. 2018;73(5):467-72.

14. van Nijnatten TJA, et al. Routine use of standard breast MRI compared to axillary ultrasound for differentiating between no, limited and advanced axillary nodal disease in newly diagnosed breast cancer patients. Eur J Radiol. 2016:85(12):2288-94

15. Zhou P, et al. Axillary lymph node metastasis detection by magnetic resonance imaging in patients with breast cancer: a meta-analysis. Thoracic Cancer. 2018;9(8):989-96.

16. Mann RM, Cho N, Moy L. Breast MRl: state of the art. Radiology. 2019;292(3):520-36.

17. LiY, et al. Computed tomography and magnetic resonance imaging evaluation of pelvic lymph node metastasis in bladder cancer. Chin J Cancer. 2018;37(1):3

18. Hong EK, et al. CT for lymph node staging of colon cancer: not only size but also location and number of lymph node count. Abdominal Radiology. 2021:46(9):4096-105.

19. Chen J, et al. Evaluation of contrast-enhanced computed tomography (CT) and magnetic resonance imaging (MRI) in the detection of retropharyngeal lymph node metastases in nasopharyngeal carcinoma patients. Cancer Manag Res. 2020:12:1733-9.

20. Uematsu T, Sano M, Homma K. In vitro high-resolution helical CT of small axillary lymph nodes in patients with breast cancer: correlation of CT and histology. AJR Am J Roentgenol. 2001;176(4):1069-74.

21. Wahl RL, et al. Prospective multicenter study of axillary nodal staging by positron emission tomography in breast cancer: a report of the staging breast cancer with PET study group. J Clin Oncol. 2004;22(2):277-85.

22. Chen C, et al. Predictive value of preoperative multidetector-row computed tomography for axillary lymph nodes metastasis in patients with breast cancer. Front Oncol. 2019:8:666.

23. Suga $K$, et al. Breast sentinel lymph node navigation with three-dimensional interstitial multidetector-row computed tomographic lymphography. Invest Radiol. 2005;40(6):336-42.

24. Nieweg OE, Tanis PJ, Kroon BBR. The definition of a sentinel node. Ann Surg Oncol. 2001;8(6):538-41.

25. Dialani V, James DF, Slanetz PJ. A practical approach to imaging the axilla. Insights Imaging. 2015;6(2):217-29.

26. Yasuta $M$, et al. Usefulness of CT-lymphography in sentinel lymph node navigation. Int J Clin Oncol. 2014;19(3):557-62.
27. Fujita T, et al. Anatomical classification of breast sentinel lymph nodes using computed tomography-lymphography. Anat Sci Int. 2018;93(4):487-94.

28. Nakagawa M, et al. Preoperative diagnosis of sentinel lymph node (SLN) metastasis using 3D CT lymphography (CTLG). Breast Cancer. 2016:23(3):519-24.

29. Ashiba H, Nakayama R. Computerized evaluation scheme to detect metastasis in sentinel lymph nodes using contrast-enhanced computed tomography before breast cancer surgery. Radiol Phys Technol. 2019;12(1):55-60.

30. Kalli S, et al. American Joint Committee on Cancer's Staging System for Breast Cancer, eighth edition: what the radiologist needs to know. Radiographics. 2018;38(7):1921-33.

31. Imai $\mathrm{N}$, et al. Strategy for the accurate preoperative evaluation of the number of metastatic axillary lymph nodes in breast cancer. Asian J Surg. 2019:42(1):228-34.

32. Nakai $\mathrm{N}$, et al. Diagnostic value of computed tomography (CT) and positron emission tomography (PET) for paraaortic lymph node metastasis from leftsided colon and rectal cancer. Asian J Surg. 2020;43(6):676-82.

33. Schwartz LH, et al. RECIST 1.1 —update and clarification: From the RECIST committee. Eur J Cancer. 2016;62:132-7.

34. LiY, et al. Risk factors analysis of pathologically confirmed cervical lymph nodes metastasis in oral squamous cell carcinoma patients with clinically negative cervical lymph node: results from a cancer center of central China. J Cancer. 2019;10(13):3062-9.

35. Li B, et al. The value of enhanced CT scanning for predicting lymph node metastasis along the right recurrent laryngeal nerve in esophageal squamous cell carcinoma. Annals of translational medicine. 2020;8(24):1632.

36. Kubota K, et al. Accuracy of multidetector-row computed tomography in the preoperative diagnosis of lymph node metastasis in patients with gastric cancer. Gastrointestinal tumors. 2017:3:163-70.

37. Lesnik D, et al. Papillary thyroid carcinoma nodal surgery directed by a preoperative radiographic map utilizing CT scan and ultrasound in all primary and reoperative patients. Head Neck. 2014;36(2):191-202.

38. Liu J, et al. Improving CT detection sensitivity for nodal metastases in oesophageal cancer with combination of smaller size and lymph node axial ratio. Eur Radiol. 2018;28(1):188-95.

39. Cho $\mathrm{N}$, et al. Preoperative sonographic classification of axillary lymph nodes in patients with breast cancer: node-to-node correlation with surgical histology and sentinel node biopsy results. Am J Roentgenol. 2009:193(6):1731-7.

40. Manca G, et al. Sentinel lymph node mapping in breast cancer: a critical reappraisal of the internal mammary chain issue. Q J Nucl Med Mol Imaging. 2014;58(2):114-26

41. Kim WH, et al. Preoperative axillary nodal staging with ultrasound and magnetic resonance imaging: predictive values of quantitative and semantic features. Br J Radiol. 2018;91(1092):20180507.

42. Marino MA, et al. Lymph node imaging in patients with primary breast cancer: concurrent diagnostic tools. Oncologist. 2020;25(2):e231-42.

43. Kuijs VJ, et al. The role of MRI in axillary lymph node imaging in breast cancer patients: a systematic review. Insights Imaging. 2015;6(2):203-15.

44. Weiss $A$, et al. Evolution in practice patterns of axillary management following mastectomy in patients with 1-2 positive sentinel nodes. Breast Cancer Res Treat. 2019:176(2):435-44.

45. DiSipio T, et al. Incidence of unilateral arm lymphoedema after breast cancer: a systematic review and meta-analysis. Lancet Oncol. 2013;14(6):500-15.

46. Jozsa $F$, et al. Is sentinel node biopsy necessary in the radiologically negative axilla in breast cancer? Breast Cancer Res Treat. 2019:177(1):1-4.

47. Fisher CS, et al. The Landmark Series: Axillary Management in Breast Cancer. Ann Surg Oncol. 2020;27(3):724-9.

48. Liang C, LiY, Luo J. A novel method to detect functional microRNA regulatory modules by bicliques merging. IEEE/ACM Trans Comput Biol Bioinform. 2016:13(3):549-56

49. Versaci M, Calcagno S, Morabito FC (2015) Image contrast enhancement by distances among points in fuzzy hyper-cubes. In: IEEE international conference. Springer International Publishing, Cham

\section{Publisher's Note}

Springer Nature remains neutral with regard to jurisdictional claims in published maps and institutional affiliations. 